\title{
Conventionally-fractionated image-guided intensity modulated radiotherapy (IG-IMRT): a safe and effective treatment for cancer spinal metastasis
}

\author{
Youling Gong ${ }^{\dagger 1,2}$, Jin Wang ${ }^{\dagger 2}$, Sen $\mathrm{Bai}^{3}$, Xiaoqin Jiang ${ }^{3}$ and Feng $\mathrm{Xu}^{* 4}$
}

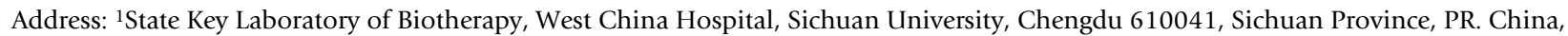
${ }^{2}$ Department of Thoracic Oncology, Tumor Center, West China Hospital, Sichuan University, Chengdu 610041, Sichuan Province, PR. China, ${ }^{3}$ Radiation\&Physics Center, Tumor Center, West China Hospital, Sichuan University, Chengdu 610041, Sichuan Province, PR. China and ${ }^{4}$ Department of Abdominal Oncology, Tumor Center, West China Hospital, Sichuan University, Chengdu 610041, Sichuan Province, PR. China

Email: Youling Gong - gongyouling@gmail.com; Jin Wang - jinwang593@yahoo.com.cn; Sen Bai - tonybaisen@yahoo.com.cn; Xiaoqin Jiang - jiangkx@yahoo.com.cn; Feng Xu* - fengxuster@gmail.com

* Corresponding author †Equal contributors

\section{Published: 22 April 2008}

Radiation Oncology 2008, 3:11 doi:10.1186/1748-717X-3-11
Received: 27 November 2007

Accepted: 22 April 2008

This article is available from: http://www.ro-journal.com/content/3/I/II

(C) 2008 Gong et al; licensee BioMed Central Ltd.

This is an Open Access article distributed under the terms of the Creative Commons Attribution License (http://creativecommons.org/licenses/by/2.0), which permits unrestricted use, distribution, and reproduction in any medium, provided the original work is properly cited.

\begin{abstract}
Background: Treatments for cancer spinal metastasis were always palliative. This study was conducted to investigate the safety and effectiveness of IG-IMRT for these patients.

Methods: 10 metastatic lesions were treated with conventionally-fractionated IG-IMRT. Daily kilovoltage cone-beam computed tomography $(\mathrm{kV}-\mathrm{CBCT})$ scan was applied to ensure accurate positioning. Plans were evaluated by the dose-volume histogram (DVH) analysis.

Results: Before set-up correction, the positioning errors in the left-right (LR), superior-inferior $(\mathrm{SI})$ and anterior-posterior $(\mathrm{AP})$ axes were $0.3 \pm 3.2,0.4 \pm 4.5$ and $-0.2 \pm 3.9 \mathrm{~mm}$, respectively. After repositioning, those errors were $0.1 \pm 0.7,0 \pm 0.8$ and $0 \pm 0.7 \mathrm{~mm}$, respectively. The systematic/random uncertainties ranged I.4-2.3/3.0-4.I before and $0.1-0.2 / 0.7-0.8 \mathrm{~mm}$ after online set-up correction. In the original IMRT plans, the average dose of the planning target volume (PTV) was 61.9 Gy, with the spinal cord dose less than $49 \mathrm{~Gy}$. Compared to the simulated PTVs based on the pre-correction CBCT, the average volume reduction of PTVs was $42.3 \%$ after online correction. Also, organ at risk (OAR) all benefited from CBCT-based set-up correction and had significant dose reduction with IGRT technique. Clinically, most patients had prompt pain relief within one month of treatment. There was no radiation-induced toxicity detected clinically during a median follow-up of 15.6 months.
\end{abstract}

Conclusion: IG-IMRT provides a new approach to treat cancer spinal metastasis. The precise positioning ensures the implementation of optimal IMRT plan, satisfying both the dose escalation of tumor targets and the radiation tolerance of spinal cord. It might benefit the cancer patient with spinal metastasis. 


\section{Background}

Spine is the most common place of cancer metastasis, especially for lung cancer and breast cancer. Each year, approximately 50,000 patients with cancer develop spinal metastasis worldwide and the 5-year over-all survival rate of these patients was less than 5\% [1,2]. All together, accompanying with the improvement of therapy for malignant tumors, the overall survival time of cancer patients prolonged and the incidence of spinal metastasis was increasing gradually. Radiotherapy is the standard treatment for vertebral metastasis of patients with cancer. Reviewing the literatures, three treatments/fractions were applied clinically worldwide: $30 \mathrm{~Gy} / 10$ fractions, $20 \mathrm{~Gy} / 5$ fractions and $8 \mathrm{~Gy} / 1$ fraction $[3,4]$. But all three treatments were palliative, and recurrences in pre-irradiated foci were frequent. Especially for those patients who only had vertebral metastasis with primary lesion controlled, higher dose may increase the local control and survival possibility of such patients.

To avoid radiation necrosis, the conventionally-fractionated radiotherapy always prescribed no more than $50 \mathrm{~Gy}$ on metastatic sites that were often insufficient to achieve acceptable local disease control and only inhibit tumor growth. The more conformal dose distribution of intensity-modulated radiation therapy (IMRT) may provide satisfactory dose coverage of tumor and avoid excessive radiation of surrounding normal tissue, therefore with potential advantage to achieve higher therapeutic ratio. However the vertebral metastasis was often adjacent to spinal cord and the sharp dose gradients between PTV and spinal cord requires high precision of daily positioning to guarantee implementation of IMRT. Without special techniques that allow highly accurate set-up and dose escalation, some patients who might benefit from radiotherapy may remain untreated or may be treated with doses unlikely to provide long-term local control.

So far, surgery is usually offered to patients with a reasonable life expectancy, whose spinal instability was present and was causing symptoms $[5,6]$. Surgery also has been used for more aggressive and relatively radio-resistant tumors. Also, the stereotactic radiosurgery is another choice for those patients. A few study reported that the single- or hypo-fractionated radiosurgery had the promising results in the treatment for cancer spinal metastasis [7$10]$. But in practice, the treatment failures were still common $[11,12]$.

To date, no ideal treatment could be prescribed for these cancer patients. The newly developed Elekta Synergy ${ }^{\mathrm{TM}}$ is an integrated image-guided radiotherapy (IGRT) system with the $\mathrm{kV}$-CBCT system attached to a digitalized medical linear accelerator that can provide onboard CBCT imaging of set-up errors. Thus, it had been stated as a potential treatment for cancer spinal metastasis. In this paper, we report the preliminary results of the application with this technique, giving details about the safety and effectiveness of IMRT dose escalation with IGRT for metastatic tumors of the spinal vertebra.

\section{Methods \\ Patient selection}

This study was carried out in Tumor Center at West China Hospital, Sichuan University, PR. China. Between May and November 2006, 9 previously treated cancer patients with confirmed diagnosis of $\leq 2$ spinal metastases and no other distant metastasis were recruited in this study. The basic and clinical characteristics of these patients were shown in Table 1. Each diagnosis was confirmed by computed tomography (CT), magnetic resonance imaging (MRI) or positron emission tomography-CT (PET-CT) before the treatment. And KPS scores of the patients were $\geq 80$ when admitted in our hospital, with life expectancy of more than 6 months. This study was carried out with the approval of West China Hospital's ethics committee.

\section{Treatment planning and evaluation}

Each patient underwent spiral CT simulation with 3-mm slice thickness with vacuum mattress (Stereotactic Body Frame, Elekta, UK) immobilization. Target volumes and normal structures were contoured by radiation physicians. The gross target volume (GTV) represented areas at cancer metastatic parts of vertebra based on pre-planning CT, MRI or PET-CT imaging. If the whole vertebra was involved, the clinical tumor volume (CTV) was defined as equal to GTV; otherwise a $10 \mathrm{~mm}$ margin around GTV was added to generate CTV. For PTV, a $3 \mathrm{~mm}$ margin was added isotropically to CTV, and the PTV was not allowed to overlap with the adjacent spinal cord but could touch it. The spinal canal was contoured as a critical structure and to extend $2 \mathrm{~cm}$ length in SI direction beyond the level of PTV, with a median length of $11.6 \mathrm{~cm}$ (range of $8.1-$ $13.4 \mathrm{~cm}$ ) in planning. Depending on the metastatic sites, the lung, right/left kidney, and liver were delineated as other OAR. All target delineations were reviewed by three physicians and brought to the final consensus. The IMRT plan was generated using 9-12 axial beam angles using aperture-based inverse planning system (PrecisePLAN Release 2.11, Electa, Sweden). A dose of 60-64 Gy was prescribed to PTV in 29-31 fractions, and the planning was to deliver the prescribed dose to at least $95 \%$ of the PTV with a dose range not exceeding $-10 \%$ and $+15 \%$ of the prescribed dose. The dose to spinal cord was restricted within $50 \mathrm{~Gy}$. The minimum segment size was $2 \mathrm{~cm}^{2}$ with a minimum of 4 monitor units (MU), a median of 43 (35-55) segments were planned. Segments were manually adjusted after aperture-based optimization to increase the dose gradient between target and OAR in 3 patients. 
Table I: Basic and clinical characteristics of the study population $(n=9)$

\begin{tabular}{ll}
\hline Age (years) & \\
$<45$ & 4 \\
$\geq 45$ & 5 \\
Gender & \\
$\quad$ Male & 4 \\
Female & 5 \\
Cancer type & \\
$\quad$ Lung cancer & 2 \\
Breast cancer & 3 \\
Colorectal cancer & 2 \\
Other cancer types & 2 \\
Spinal metastasis site $(\mathbf{n}=\mathbf{~ 1 0 )}$ & 2 \\
$\quad$ Cervical & 5 \\
Thoracic & 3 \\
Lumbar & \\
Total volume of GTV $(\mathbf{m m} 3)$ & 2 \\
$\quad \leq 20$ & 6 \\
$20 \sim 40$ & 2 \\
$\geq 40$ & \\
\hline
\end{tabular}

All plans were evaluated according to DVH analysis. The homogeneity index (HI) was defined as $\mathrm{D}_{5} / \mathrm{D}_{95}$ (minimum dose in $5 \%$ of the PTV/minimum dose in $95 \%$ of the PTV). The lower (closer to 1) the HI is, the better the dose homogeneity. Also, the conformity index (CI) was calculated as follows: $\mathrm{CI}=\mathrm{CF}$ (cover factor) $\cdot \mathrm{SF}$ (spill factor), where the $\mathrm{CF}$ was defined as the percentage of the PTV volume receiving the prescription dose and the SF as the volume of the PTV receiving the prescription dose relative to the total prescription dose-volume (see also RTOG protocol 9803). The closer the CI value to approach 1 , the better the dose conformity is.

IMRT plan was delivered with step-and-shoot technique utilizing the system's Beam Modulator ${ }^{\mathrm{rM}}$ that is an 80-leaf MLC with a leaf width of $4 \mathrm{~mm}$ (at the isocenter).

\section{KV-CBCT imaging}

Daily kV-CBCT images were acquired with the VolumeView $^{\mathrm{TM}} \mathrm{XVI}$ function. The XVI allows acquiring a series of projected images at different gantry rotation that can be reconstructed to 3-dimensional volumetric data, cut to sections and registered to input planning CT for matching. The parameters for CBCT scan were $100-120 \mathrm{kV}$, scan started from $182-260^{\circ}$ and ended at $100-180^{\circ}$ with the total imaging dose of $16 \mathrm{mGy}$ per scanning [13], utilizing medium resolution reconstruction. Each acquisition procedure (including image reconstruction) lasted 5 minutes. Daily CBCT images were registered with the planning CT using automatically bone matching (correlation coefficient algorithm, Elekta XVI software) to calculate the target deviations on the LR, SI and AP axis. The ROI for image registration was limited to the vertebrae on the level of the PTV. An action level of $2 \mathrm{~mm}$ was set for online cor- rection of translational error. Only the translational errors of the target which exceed the $2 \mathrm{~mm}$ action limit were converted to a respective shift of the treatment table by manual adjustment. Rotational set-up errors were identified but unable to correct due to limitation of couch movement. If the rotational set-up errors exceed $2^{\circ}$, patient should be re-positioned immediately. СВCT re-scan should be applied to ensure action level not exceeded. The projection of isocenter was marked on the abdominal skin of each patient to verify the maintenance of patient set-up accuracy during treatment at the first fraction, and the patient set-up remained unmovable during the whole treatment.

The positioning errors were analyzed as described previously [14]: The mean of all displacements and the standard deviation (SD) of all displacements of the whole group of patients were calculated. For each patient individually the mean (systematic error) and standard deviation (random error) of all errors were calculated. The systematic uncertainty $\Sigma$ is defined as the standard deviation of the systematic errors. The root-mean-square of the random errors was calculated as $\sigma$. Errors were calculated separately for all three axes (LR, SI and AP).

\section{Simulation of observed patient set-up errors}

According to Yan et al [15], PTV margin can be designed based on a large confidence level $(\geq 98 \%)$ with a simple recipe of $2.27 \times \mathrm{SD}$. The margins based on initial set-up errors and post-correction errors were thus calculated. Then the calculated PTV margin at initial setup was added to CTV in three directions in each IMRT plans respectively, to generate another PTV (PTV $\left.{ }_{\text {pre }}\right)$ when no online correction was applied. To simulate the impact of online correction on dosimetry, the isocenter of the original IMRT plan was shifted towards each OAR with a magnitude that was equal to the difference between the calculated pre-correction margin and actual applied margin $(3 \mathrm{~mm})$. The dosevolume parameters of OARs of the original and simulated IMRT plans were then compared.

\section{Follow-up}

Chemotherapy was prescribed after IG-IMRT. And patients were seen 1 month, and every 3 months after treatment. The $100 \mathrm{~mm}$ Visual Analog Scale (VAS) measure was used to evaluate the pain of these patients. The radiation-induced toxicities were assessed with RTOG criteria [16]. The median follow-up of the study patients was 15.6 months (range of 11-19 months).

\section{Results}

In treatment planning, the average dose which the PTVs received was $61.9 \mathrm{~Gy}$, with the maximum dose of $64.6 \mathrm{~Gy}$ and the minimum dose of $58.7 \mathrm{~Gy}$ (Figure 1). The average level of the maximum dose which the adjacent cord 


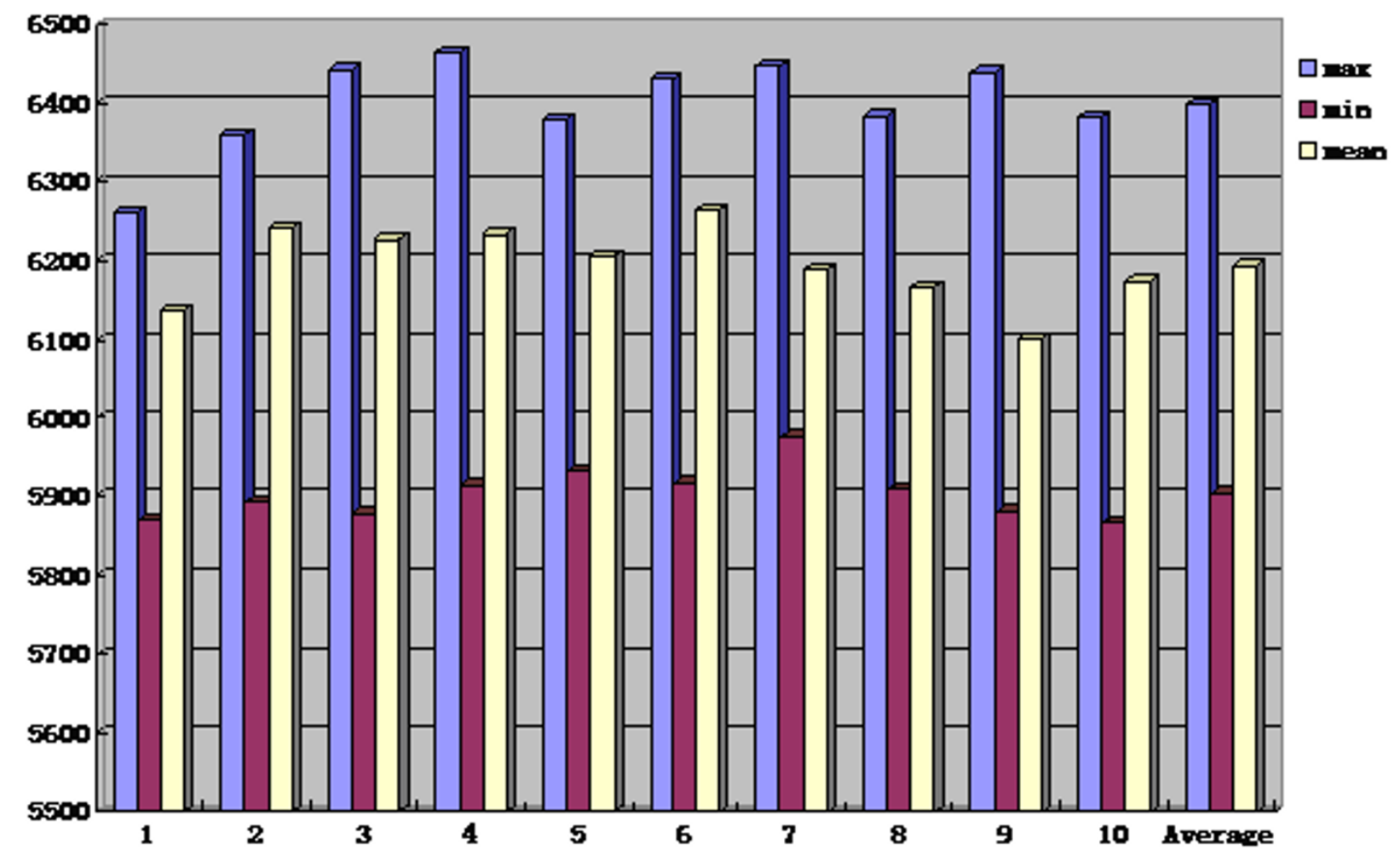

Figure I

The maximum, minimum and mean dose of the 10 metastatic lesions (PTV) in treatment plans and the average level.
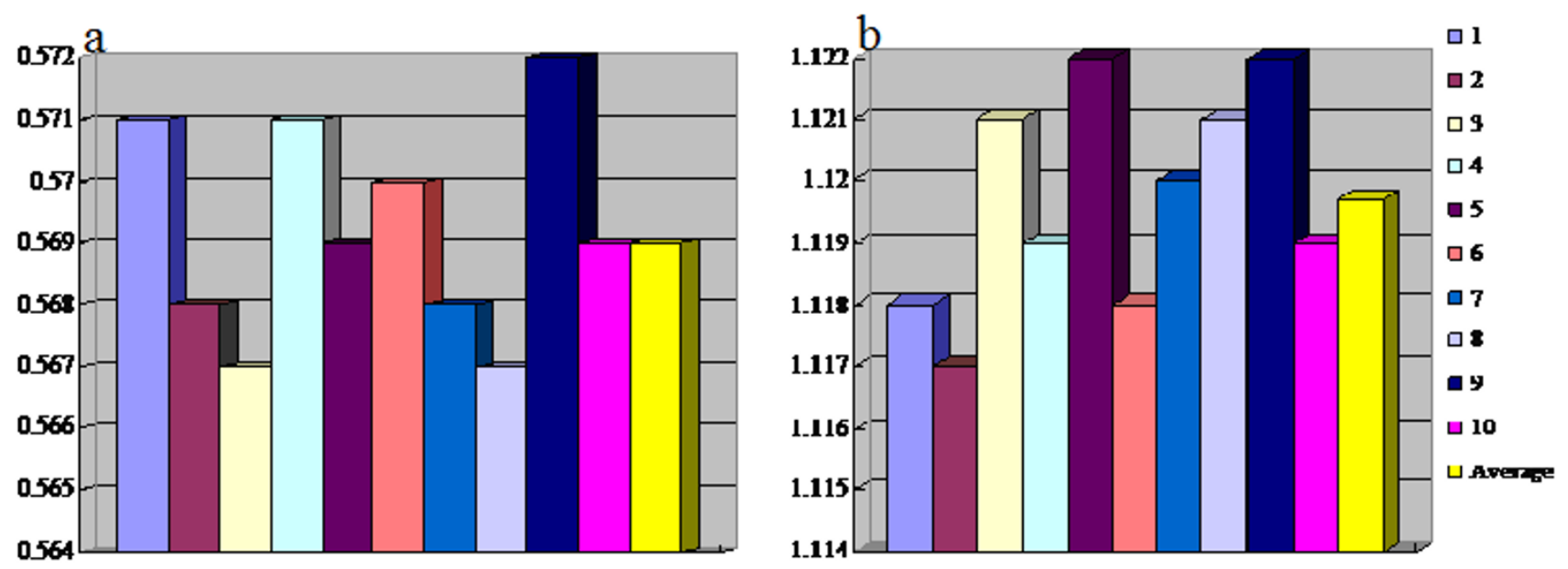

Figure 3

The homogeneity index/dose conformity index and the average level in treatment plans $(1,2,3 . .10$ represented the number of the IMRT plans, respectively). 


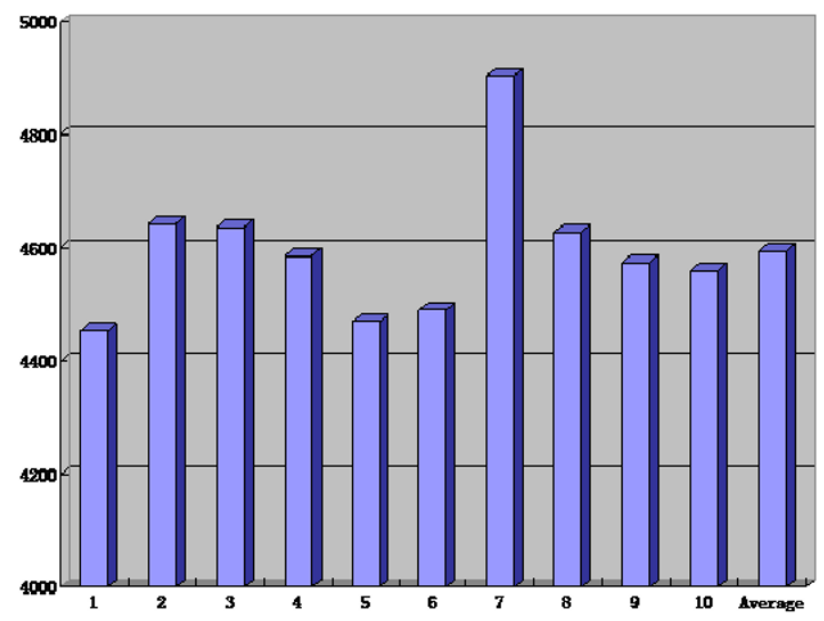

Figure 2 The maximum dose of the spinal cord in treatment plans and the average level.

received was 45.9 Gy, with a range of 44.5-49.0 Gy (Figure 2). Based on the DVH analysis, the average CI was 0.569 , with a range of $0.567-0.572$ (Figure 3 ). For HI, the maximum and the minimum values were 1.122 and 1.117 respectively, with an average value of 1.12 (Figure 3). A representative IMRT plan with radiation isodose curves was shown in Figure 4. The PTV (red region) was covered by the $95 \%$ curve ( $58.5 \mathrm{~Gy}$, the green line) of the prescription dose (60 Gy), and the curve of $47 \mathrm{~Gy}$ touched the adjacent cord.

As shown in Table 2, both systematic $(\Sigma)$ and random $(\sigma)$ uncertainties were markedly reduced after online correction which ranged $0.1-0.2 / 0.7-0.8 \mathrm{~mm}$ after correction compared to $1.4-2.3 / 3.0-4.1 \mathrm{~mm}$ before correction. And the group mean $(\mathrm{M})$ of the setup errors were small both before and after correction.

According to the calculated pre-correction margins (2.27 $\times$ SD) shown in Table 2, the volume of the actual PTV $\left(\mathrm{PTV}_{\text {real }}\right)$ in the applied IMRT plans and simulated PTV were shown in Table 3 in details. The average volume of PTV $_{\text {real }}$ and PTV pre $_{\text {was }} 77.1$ and $133.7 \mathrm{~cm}^{3}$ respectively; with an average reduction of $42.3 \%$ after online correction. The impact of translational shift of treatment isocenter towards each OAR on the dose-volume parameters was shown in Table 4. More notably, the average reduction in dose-volume parameters of OAR from PTV pre $_{\text {e }}$ PTV $_{\text {real }}$ were $14.8 \%, 10.7 \%$ and $14.5 \%$ in the mean dose, $\mathrm{V}_{20}$ and $\mathrm{V}_{12.5}$ of the lungs; $19.9 \%, 33.3 \%, 29.6 \%$ and $21.1 \%$ in the mean dose, $\mathrm{V}_{30}, \mathrm{~V}_{20}$ and $\mathrm{V}_{10}$ of the liver; $21.9 \%, 42.9 \%, 23.8 \%$ and $20.5 \%$ in the mean dose, $\mathrm{V}_{30}$, $\mathrm{V}_{20}$ and $\mathrm{V}_{10}$ of the right/left kidney; $28.2 \%$ and $16.7 \%$ in the maximum dose and $\mathrm{D}_{5}$ spine (maximum dose in $5 \%$ volume of the spinal cord) of spinal cord, respectively.

Clinically, grade $1 / 2$ acute radiation-induced skin toxicity was observed during treatment, and the majority of patients had prompt pain relief within 4 weeks of treatment. According to their VAS score, the average level was $83 \mathrm{~mm}$ (range, 70-90 $\mathrm{mm}$ ) at the baseline. 4 weeks after IG-IMRT, the average score decreased to $52 \mathrm{~mm}$, with a range of $40-62 \mathrm{~mm}$. And at the end of follow-up, the average VAS score of these patients was $42 \mathrm{~mm}$. 3 months after treatment, one patient developed progressive metastasis in the brain, and one developed liver metastasis, but the regions of the spine treated with IG-IMRT were clinically stable. No patient developed acute radiation-induced injury after the treatment. During follow-up, the lower extremity strength and ambulation of all patients remained stable and no patients have experienced complications as a result of the procedure.

\section{Discussion}

The irradiation tolerance of the spinal cord, the $\mathrm{TD}_{5 / 5}$, is considered to be in the range of $50 \mathrm{~Gy}$ for single daily fractions of 1.8-2.0 Gy [17]. The dose required for cure of a cancer spinal metastasis should be analogous to that of the primary site, which generally should not be less than 60 Gy (1.8-2.0 Gy/fraction) for solid tumors. Obviously the standard conventionally-fractionated $30-40$ Gy was insufficient for long-last control of the spinal metastasis, resulted in the infield failure to be $26 \%$ or more [18]. Several studies had been reported using single/hypo-fractionated radiosurgery for cancer spinal metastases [7-12]. According to the linear quadratic formula [19], the biological-effective-dose (BED) of the metastatic lesions received in these studies was between $60-153 \mathrm{~Gy}_{10}$. The clinical outcome indicated that radiosurgery was effective in the treatment of these patients, improving long-term palliation. However, the efficacy and safety of radiosurgery is limited by tumor volume and the closeness of targets to the critical organs, for larger tumors the dose is often reduced to avoid radiation-induced necrosis. Another limitation inherent of radiosurgery is that it delivers radiation over a single session and thus does not encounter multiple mitotic phases, which may spare the cells staying in the radioresistant phases and increases risk of recurrence especially with reduced dose [20]. Recently, improvement in radiation technique provides potential means of IMRT dose escalation for spinal metastasis cancer. Thus for the first time, conventionally-fractionated radiotherapy with daily $\mathrm{CBCT}$ online correction was applied for cancer spinal metastasis in this study: the BED was in a range of 97-107 $\mathrm{Gy}_{10}$ for the metastatic lesions and the irradiation dose of the spinal cord was less than 49 Gy in 29-31 fractions. Comparing to the data from radiosurgery, a therapeutic dose was prescribed for tumor 


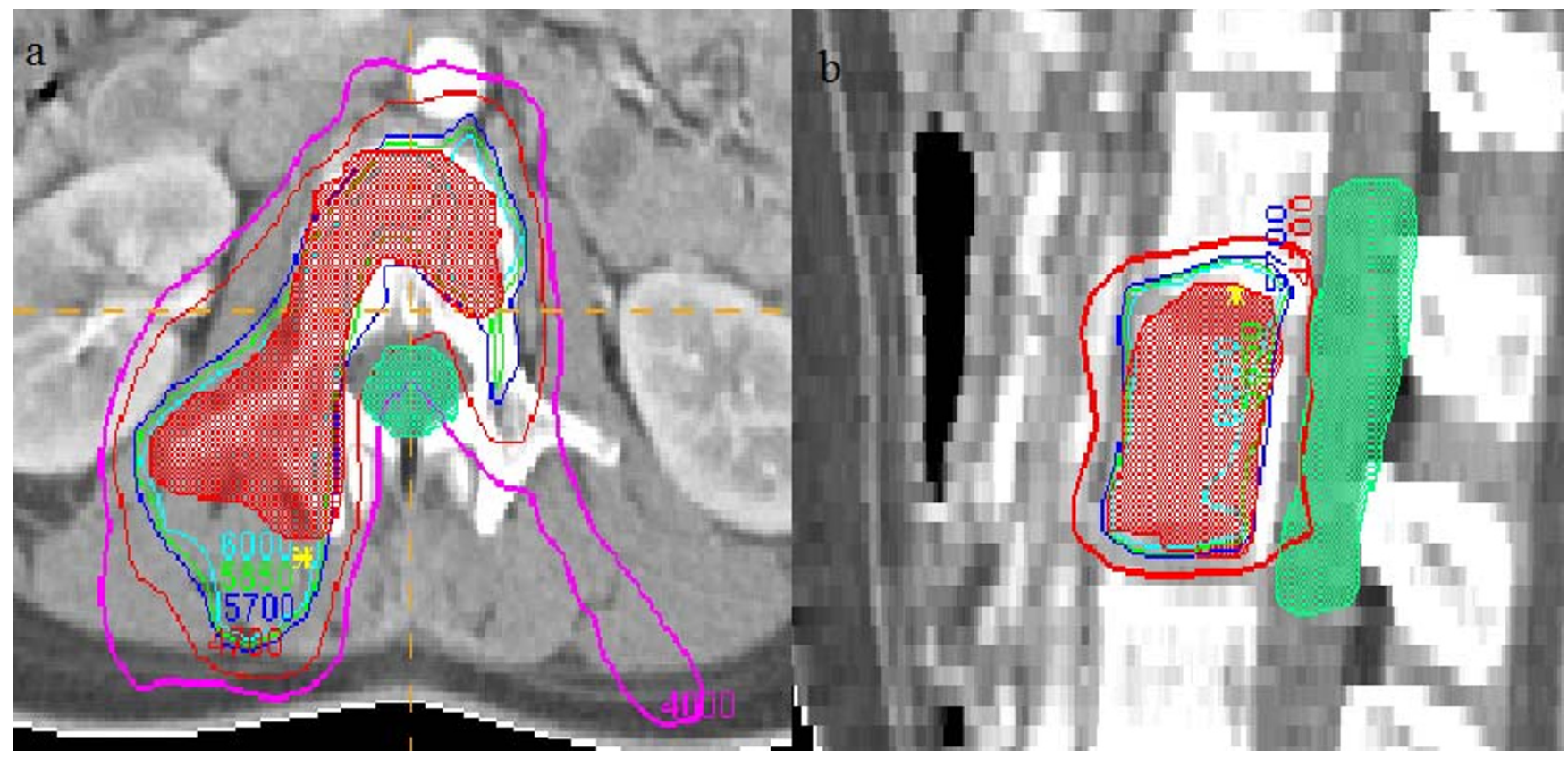

Figure 4

A representative IMRT plan with radiation isodose curves. The PTV (red region) was covered by the $95 \%$ curve of the prescription dose (the green line), and the dose of the adjacent cord was less than $49 \mathrm{~Gy}$. (a: transverse section and b: sagittal section).

target with IMRT plan, guaranteeing the irradiation tolerance of the spinal cord. Follow-up showed no patient suffering from the radiation-induced necrosis as a result of the treatment and all patients had varying degrees of pain relief. The average VAS scores of these patients were 83, 52 and $42 \mathrm{~mm}$ before, 4 weeks after IG-IMRT and at the end of follow-up, respectively. Complete pain relief was observed in 3 patients, and the remaining 6 patients were able to reduce pain medication. The result was similar with those from radiosurgery and more superior to the palliative radiotherapy in such patient. Clinically, the treatment was effective in the studied population.
Due to the steep dose gradients between metastatic lesions and spinal cord of the IMRT plan, very precise setup procedure before radiotherapy is necessary. With the application of IGRT technique, patient set-up accuracy was verified by in-room CT scanner, helical tomotherapy, orthogonal X-ray cameras, and CT on rail in radiotherapy for spinal or paraspinal cancer [12,21-24]. Basically, simply applying the patient immobilizing technique with wall laser marks on the body surface still can not fulfill the stringent target position requirement of high precision radiotherapy. In this study, daily CBCT with online correction of set-up errors before treatment was practiced to

Table 2: The positioning errors before/after (without/with) online set-up correction in the LR, SI and AP axes in this study (mm)

\begin{tabular}{|c|c|c|c|c|c|c|}
\hline & \multicolumn{2}{|c|}{ LR } & \multicolumn{2}{|c|}{ SI } & \multicolumn{2}{|c|}{ AP } \\
\hline & Before & After & Before & After & Before & After \\
\hline mean & 0.3 & 0.1 & 0.4 & 0.0 & -0.2 & 0.0 \\
\hline SD & 3.2 & 0.7 & 4.5 & 0.8 & 3.9 & 0.7 \\
\hline Range & $-12.0 \sim 13.5$ & $-2.6 \sim 1.4$ & $-17.2 \sim 16.3$ & $-2.5 \sim 1.5$ & $-12.9 \sim 10.9$ & $-1.9 \sim 1.5$ \\
\hline$\Sigma$ & 1.4 & 0.2 & 2.1 & 0.2 & 2.3 & 0.1 \\
\hline$\sigma$ & 3.0 & 0.8 & 4.I & 0.7 & 3.2 & 0.7 \\
\hline Theoretic margin & 7.4 & 1.7 & 10.2 & 1.6 & 8.8 & 1.7 \\
\hline Translational shift & \multicolumn{2}{|c|}{4.4} & \multicolumn{2}{|c|}{7.2} & \multicolumn{2}{|c|}{5.8} \\
\hline
\end{tabular}

Before: before online set-up correction; After: after online set-up correction; Theoretic margin: calculated by $2.27 \times$ SD based on a pre-selected confidence level of $98 \%$; Translational shift: translational shift of the treatment isocenter in simulated IMRT plan to cover the theoretic margin without online set-up correction in three axes, and calculated as "theoretic margin before online set-up correction-3" mm. 
Table 3: The volumes of original and simulated PTVs in this study $\left(\mathrm{cm}^{3}\right)$

\begin{tabular}{cccc}
\hline Target number & PTVreal & PTVpre & Volume reduction from PTVpre to PTVreal (\%) \\
\hline 1 & 15.6 & 26.4 & $40.9 \%$ \\
2 & 94.7 & 168.6 & $43.8 \%$ \\
3 & 82.5 & 126.2 & $34.6 \%$ \\
4 & 89.3 & 150.6 & $40.7 \%$ \\
5 & 72.6 & 116.4 & $37.6 \%$ \\
6 & 18.9 & 37.2 & $49.2 \%$ \\
7 & 108.4 & 204.3 & $46.9 \%$ \\
8 & 91.2 & 155.8 & $41.4 \%$ \\
9 & 112.3 & 221.2 & $49.2 \%$ \\
10 & 85.9 & 130.6 & $34.2 \%$ \\
Average & 77.1 & 133.7 & $42.3 \%$ \\
\hline
\end{tabular}

PTVreal: actual PTV in the original IMRT plans; PTVpre: simulated PTV based on the theoretic margins in three axes without online set-up correction.

achieve the maximum accuracy and safety for the patient. The systematic/random errors at initial set-up were 1.4/ 3.0, 2.1/4.1 and 2.3/3.2 $\mathrm{mm}$ in the LR, SI and AP axes, respectively. After set-up correction, those errors were 0.2 / $0.8,0.2 / 0.7$ and $0.1 / 0.7 \mathrm{~mm}$ in the three axes respectively, indicating the role of online correction on improving positioning precision for radiotherapy of spinal metastatic cancer, thus may potentially reduce the adverse effect of set-up errors on tumor control probability and normal tissue complication probability (NTCP) in radiotherapy treatment [25].
Based on the margin-calculating recipe, a 1.7, 1.6 and 1.7 $\mathrm{mm}$ margin should be added to the CTV for generating PTV in the LR, SI and AP axes respectively with CBCT online correction, confirming that the $3 \mathrm{~mm}$ region around the GTV/CTV was enough and acceptable with CBCT-based guidance. Without online correction, the calculated margins in the three axes were 7.4, 10.2 and 8.8 $\mathrm{mm}$, respectively. In each IMRT plan, we simulated the hypothetic effects of the pre-correction positioning errors on PTV and dose-volume parameters of OAR. As shown in Table 3, the reduction of volume from the pre-correction $\mathrm{PTV}_{\text {pre }}$ to the PTV $\mathrm{Pral}_{\text {ral }}$ with online correction was considera-

Table 4: Average normal tissue dose-volume parameters based on PTVpre and PTVreal in each original and simulated IMRT plans

\begin{tabular}{|c|c|c|c|}
\hline Normal tissue parameter & Average parameters based on PTVpre & $\begin{array}{c}\text { Average parameters based on } \\
\text { PTVreal }\end{array}$ & $\begin{array}{c}\text { Average parameters reductions from } \\
\text { PTV pre to PTVreal (\%) }\end{array}$ \\
\hline \multicolumn{4}{|l|}{ Lung $(n=4)$} \\
\hline Maximum dose & $57.3 \mathrm{~Gy}$ & $55.2 \mathrm{~Gy}$ & 3.7 \\
\hline Average dose & $10.8 \mathrm{~Gy}$ & $9.2 \mathrm{~Gy}$ & 14.8 \\
\hline V20 & $12.2 \%$ & $10.9 \%$ & 10.7 \\
\hline VI2.5 & $23.5 \%$ & $20.1 \%$ & 14.5 \\
\hline \multicolumn{4}{|l|}{ Liver $(n=4)$} \\
\hline Maximum dose & $58.6 \mathrm{~Gy}$ & 56.l Gy & 4.3 \\
\hline Average dose & $14.6 \mathrm{~Gy}$ & II.7 Gy & 19.9 \\
\hline V30 & $12 \%$ & $8 \%$ & 33.3 \\
\hline $\mathrm{V} 20$ & $27 \%$ & $19 \%$ & 29.6 \\
\hline V10 & $38 \%$ & $30 \%$ & 21.1 \\
\hline \multicolumn{4}{|l|}{ kidney $(n=4)$} \\
\hline Maximum dose & $60.2 \mathrm{~Gy}$ & $57.8 \mathrm{~Gy}$ & 4.0 \\
\hline Average dose & $14.6 \mathrm{~Gy}$ & II.4 Gy & 21.9 \\
\hline V30 & $7 \%$ & $4 \%$ & 42.9 \\
\hline $\mathrm{V} 20$ & $21 \%$ & $16 \%$ & 23.8 \\
\hline VIO & $39 \%$ & $31 \%$ & 20.5 \\
\hline \multicolumn{4}{|l|}{ Cord $(n=10)$} \\
\hline Maximum dose & $68.4 \mathrm{~Gy}$ & 49.1 Gy & 28.2 \\
\hline Average dose & $34.3 \mathrm{~Gy}$ & $31.2 \mathrm{~Gy}$ & 9.1 \\
\hline D5spine & $54.4 \mathrm{~Gy}$ & $45.3 \mathrm{~Gy}$ & 16.7 \\
\hline
\end{tabular}

PTV pre: simulated PTV based on the theoretic margins in the three axes without online set-up correction; PTVreal: actual PTV in the original IMRT plans; D5spine: maximum dose in $5 \%$ volume of the spinal cord. 


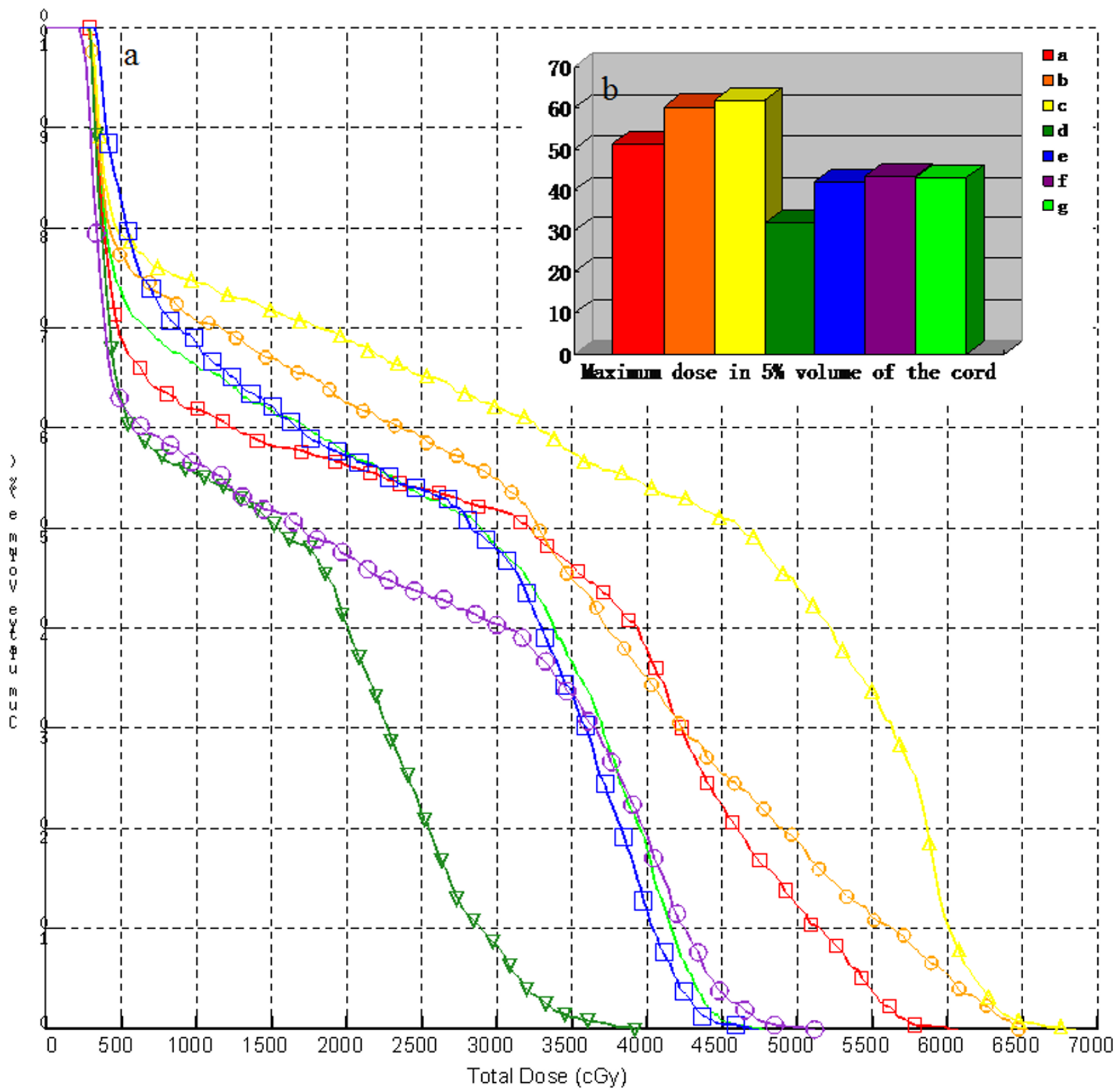

Figure 5

Comparison of the simulated effects of the positioning errors with/without CBCT-based online set-up correction in the LR, SI and AP axes on the irradiation dose of the spinal cord with the actual plan (red and orange line: isocenter moving left/right in LR axis, yellow and deep green lines: isocenter moving superior/inferior in SI axis, blue and purple lines: isocenter moving anterior/posterior in AP axis, respectively; and the green line was the actual DVH of the cord). (a: the simulated and actual DVHs of the cord and $\mathrm{b}$ : the simulated and actual maximum dose of $5 \%$ volume of the cord).

ble, with an average level of $42.3 \%$. Also, the translational shift of isocenter towards each OAR had significant impact on the dose-volume parameters of these organs. Depending on the target location, there were 4 targets related to lung, 4 targets related liver and right/left kidney, and 10 targets related to spinal cord. The dose-volume parameters of each OAR were reduced to varying degrees.
The dose reductions could translate clinically into a lower probability of treatment toxicity, as well as a potential increase in the number of patients that might be eligible for IG-IMRT or concurrent chemoradiotherapy.

The spinal cord was the key OAR in this study. The isocenter was shifted in the six directions (moving left/right, 
inferior/superior, and anterior/posterior in LR, SI and AP axes) respectively to simulate the impact of pre-correction margin on the dose-volume parameters of the spinal cord. Figure 5 showed the simulated and original DVH of the spinal cord in one IMRT plan (the same patient as Figure 4 represented). The position errors in SI axes had little impact on the irradiation dose of the cord. As well, it indicated that the $\mathrm{D}_{5}$ spine changed significantly, if position errors occurred towards the cord in LR and AP axis, respectively. Most significantly, the posterior shift towards the cord resulted in a maximum dose of $68 \mathrm{~Gy}$ to the cord. Comparing to the results reported by Guckenberger et al [26], our study suggested that without the СВCT online guidance, the IMRT plan could not be applied successfully in such patients.

Although only the inter-fractional setup errors was taken into account as the major source of uncertainties to affect the accuracy of IMRT dose delivery in this study, there was another important factor which also contribute to the dose delivery accuracy: movement of the target and spinal cord during treatment (intra-fraction variation). First, CTV for the paraspinal lesions was assumed to be fixed to the vertebrae and the intra-treatment motion of the target would be equivalent to the motion of the spinal column. Data from literatures have confirmed that in conformal radiotherapy, intra-fraction organ/target motion can be achieved in the range of $1 \mathrm{~mm}$ with proper immobilization $[21,27]$. Second, Cai et al found that the spinal cord motion during normal breathing was typically within 0.5 $\mathrm{mm}$ by dynamic MRI (dMRI), and partly stated that the spinal cord was almost immovable during breathing [28]. Third, studies in Massachusetts General Hospital and Memorial Sloan-Kettering Cancer Center indicated that the effects of intra-fraction organ motion on IMRT dose delivery were ignorable in a typical treatment with 30 fractions in breast and pulmonary radiotherapy [29,30]. Obviously, for the more fixed OAR (spinal cord) in the vacuum mattress, the effects of intra-fraction organ motion would be more minimal in this conventionallyfractionated IMRT therapy. In addition, all target delineations were reviewed by three physicians together, diminishing the impact of the delineation-induced variation on the geometrical accuracy in conformal radiotherapy [3133] as far as possible. Consequently, as discussed previously, the precise patient set-up with СВСТ online correction was the minimal requirement and meaningful factor in dose delivery accuracy of IG-IMRT in this study.

Limitation in this study should be addressed here. The position errors should include not only the translational set-up errors, but also the rotational errors, which may have effect on the accuracy of dose delivery. A few studies evaluated the rotational set-up errors in conformal radiotherapy for spinal diseases $[26,34]$. Due to the limitation of the treatment couch, patient in our study should be repositioned if the rotational set-up errors exceeded $2^{\circ}$. So, the rotational set-up errors and their impact on IMRT dose delivery had not been evaluated in this study.

\section{Conclusion}

Therefore, this study presented the preliminary data to demonstrate the safety and effectiveness of this technique in treatment of patients with cancer spinal metastasis. These results are encouraging. Although the studied sample size was somewhat small and with the limitation mentioned above, it still was a hopeful progress in radiation therapy for patient with cancer. As a result, the application of conventionally-fractionated IG-IMRT has the potential to improve the clinical outcome of the patients with cancer spinal metastasis.

\section{Competing interests}

The authors declare that they have no competing interests.

\section{Authors' contributions}

YG and JW contributed equally in design of the study, collection of data and drafting the manuscript; $\mathrm{SB}$ and $\mathrm{XJ}$ worked on analysis of data; FX provided the conception of this study and the final approval of the version to be published. And all authors read and approved the final manuscript.

\section{Acknowledgements}

We thank Dr. Xin Wang and technicians Renming Zhong, Xiaoyu Li and Yinbo $\mathrm{He}$ for their assistance in data collection.

\section{Financial supports}

This study was supported in part by Science and Technology Key Project of Sichuan Province, PR. China (Project 03SG022-008 to J.W. and 04SG022007 to F.X.).

\section{References}

I. Schmidt MH, Klimo P Jr, Vrionis FD: Metastatic spinal cord compression. J Natl Compr Canc Netw 2005, 3:7I I-7I9.

2. Steinmetz MP, Mekhail A, Benzel EC: Management of metastatic tumors of the spine: strategies and operative indications. Neurosurg Focus 200I, I I(6):e2.

3. Barton R, Robinson G, Gutierrez E, Kirkbride P, McLean M: Palliative radiation for vertebral metastases: the effect of variation in prescription parameters on the dose received at depth. Int J Radiat Oncol Biol Phys 2002, 52:1083-I091.

4. Wu JS, Wong R, Johnston M, Bezjak A, Whelan T, Cancer Care Ontario Practice Guidelines Initiative Supportive Care Group: Cancer care ontario practice guidelines initiative supportive care group: meta-analysis of dose-fractionation radiotherapy trials for the palliation of painful bone metastases. Int J Radiat Oncol Biol Phys 2003, 55:594-605.

5. Chi JH, Parsa AT: Intramedullary spinal cord metastasis: clinical management and surgical considerations. Neurosurg Clin $\mathrm{N}$ Am 2006, I 7:45-50.

6. Witham TF, Khavkin YA, Gallia GL, Wolinsky JP, Gokaslan ZL: Surgery insight: current management of epidural spinal cord compression from metastatic spine disease. Nat Clin Pract Neurol 2006, 2:87-94.

7. Bilsky MH: New therapeutics in spine metastases. Expert Rev Neurother 2005, 5:83 I-840. 
8. Ryu SI, Chang SD, Kim DH, Murphy MJ, Le QT, Martin DP, Adler JR Jr: Image-guided hypofractionated stereotactic radiosurgery to spinal lesions. Neurosurgery 200I, 49:838-846.

9. Gerszten PC, Burton SA, Belani CP, Ramalingam S, Friedland DM, Ozhasoglu C, Quinn AE, McCue KJ, Welch WC: Radiosurgery for the treatment of spinal lung metastases. Cancer 2006, 107:2653-266I.

10. Gerszten PC, Burton SA, Ozhasoglu C: CyberKnife radiosurgery for spinal neoplasms. Prog Neurol Surg 2007, 20:340-358.

11. Chang EL, Shiu AS, Mendel E, Mathews LA, Mahajan A, Allen PK, Weinberg JS, Brown BW, Wang XS, Woo SY, Cleeland C, Maor MH, Rhines LD: Phase I/II study of stereotactic body radiotherapy for spinal metastasis and its pattern of failure. J Neurosurg Spine 2007, 7:151-160.

12. Ryu S, Rock J, Rosenblum M, Kim JH: Patterns of failure after single-dose radiosurgery for spinal metastasis. J Neurosurg 2004, I0 I(Suppl 3):402-405.

13. Clinical user manual for XVI, Elekta synergy, ELEKTA. 2005, R 3.5:A-7.

14. van Herk M: Errors and margins in radiotherapy. Semin Radiat Oncol 2004, 14:52-64.

15. Yan D, Lockman D, Martinez A, Wong J, Brabbins D, Vicini F, Liang J, Kestin L: Computed tomography guided management of interfractional patient variation. Semin Radiat Oncol 2005, 15:168-179.

16. Cox JD, Stetz J, Pajak TF: Toxicity criteria of the Radiation Therapy Oncology Group (RTOG) and the European Organization for Research and Treatment of Cancer (EORTC) [Editorial]. Int / Radiat Oncol Biol Phys 1995, 3 I:134I-1346.

17. Marcus RB Jr, Million RR: The incidence of myelitis after irradiation of the cervical spinal cord. Int J Radiat Oncol Biol Phys 1990, 19:3-8.

18. Rades D, Stalpers LJ, Veninga T, Schulte R, Hoskin PJ, Obralic N, Bajrovic A, Rudat V, Schwarz R, Hulshof MC, Poortmans P, Schild SE: Evaluation of five radiation schedules and prognostic factors for metastatic spinal cord compression. J Clin Oncol 2005, 23:3366-3375.

19. Fowler JF: The linear quadratic formula and progress in fractionated radiotherapy. $\mathrm{Br} /$ Radiol 1989, 62:679-694.

20. Giller CA, Berger BD: New frontiers in radiosurgery for the brain and body. Proc (Bayl Univ Med Cent) 2005, I 8(4):3II-3I9.

21. Yamada Y, Lovelock DM, Yenice KM, Bilsky MH, Hunt MA, Zatcky J, Leibel SA: Multifractionated image-guided and stereotactic intensity-modulated radiotherapy of paraspinal tumors: a preliminary report. Int I Radiat Oncol Biol Phys 2005, 62:53-6I.

22. Yenice KM, Lovelock DM, Hunt MA, Lutz WR, Fournier-Bidoz N, Hua CH, Yamada J, Bilsky M, Lee H, Pfaff K, Spirou SV, Amols HI: CT image-guided intensity-modulated therapy for paraspinal tumors using stereotactic immobilization. Int J Radiat Oncol Biol Phys 2003, 55:583-593.

23. Mahan SL, Ramsey CR, Scaperoth DD, Chase DJ, Byrne TE: Evaluation of image-guided helical tomotherapy for the retreatment of spinal metastasis. Int J Radiat Oncol Biol Phys 2005, 63:1576-1583

24. Chang EL, Shiu AS, Lii MF, Rhines LD, Mendel E, Mahajan A, Weinberg IS, Mathews LA, Brown BW, Maor MH, Cox JD: Phase I clinical evaluation of near-simultaneous computed tomographic image-guided stereotactic body radiotherapy for spinal metastases. Int J Radiat Oncol Biol Phys 2004, 59: I 288- 1294.

25. van Herk M, Remeijer P, Lebesque JV: Inclusion of geometric uncertainties in treatment plan evaluation. Int J Radiat Oncol Biol Phys 2002, 52: I 407-I 422.

26. Guckenberger M, Meyer J, Wilbert J, Baier K, Bratengeier K, Vordermark $D$, Flentje M: Precision required for dose-escalated treatment of spinal metastases and implications for image-guided radiation therapy (IGRT). Radiother Oncol 2007, 84:56-63.

27. Guckenberger M, Meyer J, Wilbert J, Baier K, Sauer O, Flentje M: Precision of image-guided radiotherapy (IGRT) in six degrees of freedom and limitations in clinical practice. Strahlenther Onkol 2007, | 83:307-3|3.

28. Cai J, Sheng K, Sheehan JP, Benedict SH, Larner JM, Read PW: Evaluation of thoracic spinal cord motion using dynamic MRI. Radiother Oncol 2007, 84:279-282.

29. Bortfeld T, Jokivarsi K, Goitein M, Kung J, Jiang SB: Effects of intrafraction motion on IMRT dose delivery: statistical analysis and simulation. Phys Med Biol 2002, 47:2203-2220.
30. Chui CS, Yorke E, Hong L: The effects of intra-fraction organ motion on the delivery of intensity-modulated field with a multileaf collimator. Med Phys 2003, 30:1736-1746.

3I. Leunens G, Menten J, Weltens C, Verstraete J, Schueren E van der: Quality assessment of medical decision making in radiation oncology: variability in target volume delineation for brain tumors. Radiother Oncol 1993, 29:169-175.

32. Fiorino C, Reni M, Bolognesi A, Cattaneo GM, Calandrino R: Intraand inter-observer variability in contouring prostate and seminal vesicles: implications for conformal treatment planning. Radiother Oncol 1998, 47:285-292.

33. Dubois DF, Prestidge BR, Hotchkiss LA, Prete J], Bice WS Jr: Intraobserver and interobserver variability of MR imagingand CT-derived prostate volumes after transperineal interstitial permanent prostate brachytherapy. Radiology 1998, 207:785-789.

34. Onimaru R, Shirato H, Aoyama H, Kitakura K, Seki T, Hida K, Fujita K, Kagei K, Nishioka T, Kunieda T, Iwasaki Y, Miyasaka K: Calculation of rotational setup error using the real-time tracking radiation therapy (RTRT) system and its application to the treatment of spinal schwannoma. Int J Radiat Oncol Biol Phys 2002, 54:939-947.

Publish with Bio Med Central and every scientist can read your work free of charge

"BioMed Central will be the most significant development for disseminating the results of biomedical research in our lifetime. "

Sir Paul Nurse, Cancer Research UK

Your research papers will be:

- available free of charge to the entire biomedical community

- peer reviewed and published immediately upon acceptance

- cited in PubMed and archived on PubMed Central

- yours - you keep the copyright

Submit your manuscript here:

http://www.biomedcentral.com/info/publishing_adv.asp
BioMedcentral 